\title{
Detection of Impulse Radio Ultra-Wideband Signals using Recursive Transmitted Reference Receivers
}

\author{
Muhammad Gufran Khan, Jörgen Nordberg, and Ingvar Claesson \\ Department of Signal Processing, School of Engineering, \\ Blekinge Institute of Technology, SE-372 25 Ronneby, Sweden \\ Email: \{Muhammad.Gufran.Khan, Jorgen.Nordberg, Ingvar.Claesson\}@bth.se
}

\begin{abstract}
For the detection of impulse radio (IR) UWB signals, complex RAKE receiver or low-complexity transmitted reference (TR) autocorrelation receivers can be used. However, there is a performance loss associated with the low-complexity TR scheme. This paper proposes recursive structures of the conventional TR and averaged TR schemes to improve the detection performance of IR-UWB signals. The performance of proposed schemes is evaluated over the standard IEEE 802.15.4a multipath channels. The performance is compared with conventional TR receivers in terms of uncoded bit-error-rate (BER), assuming that the channel is quasi-static. For averaged and recursive averaged TR schemes, the TR sequence is also slightly modified. The simulation results validate that the proposed schemes has better performance by about $2 \mathrm{~dB}$ than the conventional TR and averaged TR receivers.
\end{abstract}

\section{INTRODUCTION}

Ultra-wideband (UWB) radio is emerging worldwide as a particularly appealing transmission technique for applications requiring either high bit rates over short ranges or low bit rates over medium-to-long ranges [1]. The impulse radio UWB offers some unique qualities such as high-resolution, low power spectral density and wide bandwidth which makes them suitable for diverse applications such as wireless personal area networks (WPANs) and wireless sensor networks.

The impulse radio UWB systems utilize very narrow pulses for the transmission. The sub-nanosecond nature of these pulses provides rich multipath diversity at the receiver as a large number of (on the order of hundred) multipath components (MPCs) can be resolved. The multipath diversity available at the receiver can be exploited using a RAKE structure [2]. Since the number of resolved MPCs is very high, RAKE receiver needs a large number of correlators (fingers) [3]. Providing a receiver with a large number of correlators, however, increases the receiver complexity [4]. Furthermore, a RAKE receiver needs to estimate the channel impulse response in order to obtain the correct RAKE weights [5].

Alternatively, non-coherent autocorrelation receiver can capture the entire received signal energy for slowly varying channels without requiring channel estimation [6]. Transmitted reference scheme that has recently gained significant attention, is one sub-optimum autocorrelation receiver scheme for the demodulation of IR-UWB signals [6], [7]. Transmitted reference communication systems operate by transmitting a pair of unmodulated and modulated signals and employing the former to demodulate the latter [7]. Since the reference signal and data signal are transmitted within the coherence time of the channel, it is assumed that the channel responses to the two signals are the same [8]. One drawback of TR scheme is a loss of at least $6 \mathrm{~dB}$ compared to an ideal matched filter receiver, $3 \mathrm{~dB}$ due to noise contained within the reference pulse and $3 \mathrm{~dB}$ due to usage of two pulses per bit, instead of one [9].

In the literature, different transmitted reference receiver schemes have been described with the aim to achieve a lowcomplexity detection. In [6], an averaged TR (ATR) system which performs averaging of all the reference pulses in a previous symbol interval has been proposed to remedy the problem of noisy reference pulse. The suppression of noise by averaging previously received reference pulses requires the implementation of precise delays which may be burdensome [6]. In [8], optimal and suboptimal UWB TR receivers are analyzed and a differential TR system is presented. A differential and TR detector, and their iterative solutions are considered and analyzed in [10]. A decision directed autocorrelation receiver and its recursive solution is proposed in [11] for pulsed ultra-wideband systems. The decision directed scheme decreases the signaling overhead but relies on the past symbol decisions.

In this paper, a recursive TR (R-TR) and a recursive averaged TR (R-ATR) receiver scheme is investigated and proposed with the aim to improve the performance of the conventional IR-UWB TR schemes. The R-TR and R-ATR structures estimate the reference template for the correlator recursively from the received reference pulses. The receiver has still low-complexity as the correlation can be performed in the analog domain avoiding the high sampling requirements. The comparison of the proposed receivers has been performed with the conventional TR and ATR receivers. The simulation results validate that the proposed schemes exhibit a performance gain over the conventional TR receivers.

The rest of the paper is outlined as follows. In section II, the signal model of a typical TR IR-UWB system is described and the UWB channel model used for the performance evaluation of the system is discussed. Section III presents the conventional and proposed TR receiver structures. The performance evaluation and the results are discussed in section IV and finally the conclusions are presented in section V. 


\section{Signal Model}

The transmitted signal of a IR-UWB system using binary antipodal modulation and TR signaling can be written as

$$
\begin{aligned}
s(t)= & \sqrt{\frac{E_{b}}{2 N_{f}}} \sum_{j=-\infty}^{\infty}\left[p\left(t-j T_{f}\right)\right. \\
& \left.+b_{\left\lfloor j / N_{f}\right\rfloor} p\left(t-j T_{f}-T_{d}\right)\right],
\end{aligned}
$$

where $p(t)$ is the transmitted UWB pulse, $E_{b}$ is the symbol energy, $T_{f}$ is the frame duration. Each transmitted bit contains $N_{f}$ successive frames and each frame contains two pulses separated by a fixed delay of duration $T_{d}$. Thus, one binary information symbol $b_{\left\lfloor j / N_{f}\right\rfloor} \in\{1,-1\}$ is transmitted by a stream of $2 N_{f}$ pulses. In Fig. 1, the block diagram of the simplified TR transmitter introduced by Hoctor and Tomlinson [7] is shown. In Fig. 2(a), an example of the transmitted sequence for TR and R-TR receiver schemes is shown. Similarly, Fig. 2(b) represents the transmitted sequence of pulses which has been slightly modified for ATR and R-ATR receiver schemes.

The signal is further transmitted over a multipath channel. The channel model used for the evaluation of system performance is proposed by IEEE 802.15.4a group for sensor networks and similar devices with data rates between $1 \mathrm{kbit} / \mathrm{s}$ and several Mbit/s for different office, residential and industrial scenarios. It is a modified version of the Saleh-Valenzuela (SV) model [12] and considers that the multipath components arrive in clusters. The impulse response of the general S-V model is given as in [12],

$$
h(t)=\sum_{l=0}^{L-1} \sum_{k=0}^{K-1} \alpha_{k, l} \delta\left(t-T_{l}-\tau_{k, l}\right),
$$

where $\alpha_{k, l}$ is the tap weight of the $k$ th ray (path) in the $l$ th cluster, $T_{l}$ is the arrival time of the lth cluster and $\tau_{k, l}$ is the arrival time of the $k$ th ray in the $l$ th cluster. The key features of the IEEE 802.15.4a model include a mixed Poisson distribution for ray arrival times, possible delay dependence of cluster decay times, frequency dependence of the path loss and Nakagami $-m$ distribution for small scale fading statistics [13]. The impulse response of the simplified version of the $\mathrm{S}-\mathrm{V}$ model can be written as

$$
h(t)=\sum_{l=0}^{L-1} \alpha_{l} \delta\left(t-\tau_{l}\right)
$$

where $\alpha_{l}$ are the channel tap weights, $L$ is the number of multipath components and $\tau_{l}$ is the delays associated with $l$ th multipath components. The received signal after passing through the simplified multipath channel can be expressed as

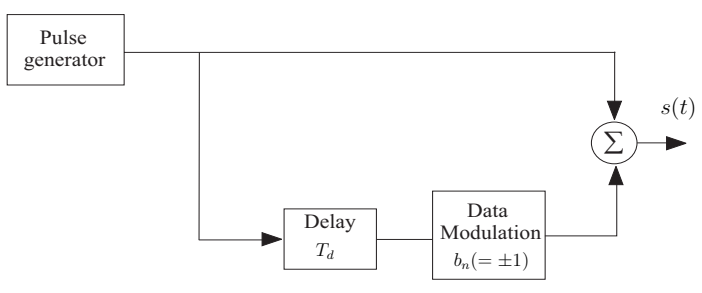

Fig. 1. Block diagram of the simplified TR transmitter (for both signaling schemes shown in Fig.2).

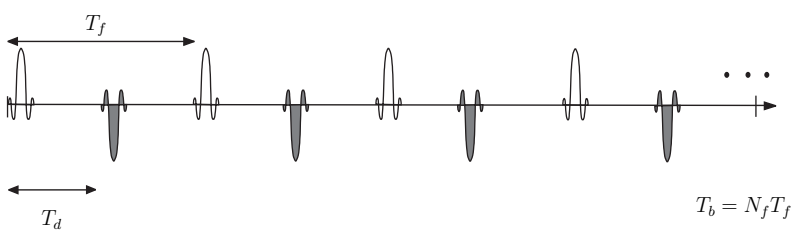

(a)

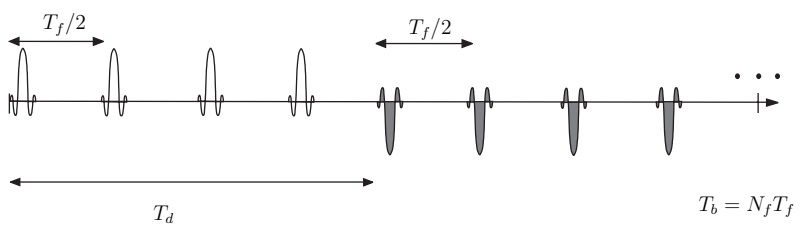

(b)

Fig. 2. An example of the transmitted sequence (a) for TR and R-TR scheme, (b) for ATR and R-ATR scheme, $N_{f}=4$ in these figures and the data pulses are shown filled.

$$
\begin{aligned}
r(t)= & \sqrt{\frac{E_{b}}{2 N_{f}}} \sum_{j=-\infty}^{\infty} \sum_{l=0}^{L-1}\left[\alpha_{l} p\left(t-j T_{f}-\tau_{l}\right)\right. \\
& \left.+b_{\left\lfloor j / N_{f}\right\rfloor} \alpha_{l} p\left(t-j T_{f}-T_{d}-\tau_{l}\right)\right]+n(t) \\
= & \sqrt{\frac{E_{b}}{2 N_{f}}} \sum_{j=-\infty}^{\infty}\left[g\left(t-j T_{f}\right)\right. \\
& \left.+b_{\left\lfloor j / N_{f}\right\rfloor} g\left(t-j T_{f}-T_{d}\right)\right]+n(t),
\end{aligned}
$$

where $g(t)$ is interpreted as the aggregate channel after convolving with the transmitted pulse, $g(t)=p(t) * h(t)$, i.e.,

$$
g(t)=\sum_{l=0}^{L-1} \alpha_{l} p\left(t-\tau_{l}\right),
$$

where $n(t)$ is a Gaussian process with zero mean and variance $\sigma_{n}^{2}$. The duration of $g(t)$ is defined as $T_{g}=T_{p}+T_{m d s}$, where $T_{p}$ is the pulse duration, $T_{m d s}$ is the maximum delay spread of the channel. In practice, the interference between reference and modulated pulses can be avoided by keeping the delay $T_{g} \leq T_{d}$.

\section{ReCEIVER Structures}

The received signal is passed through a filter which is matched to the transmitted pulse. For simplicity, it is assumed 
that the shape of the received pulse is the same as the transmitted pulse. However, the propagation and antenna effects may distort the received pulses. The output of the filter denoted by $\tilde{r}(t)$ is given as

$$
\begin{aligned}
\tilde{r}(t)= & \sqrt{\frac{E_{b}}{2 N_{f}}} \sum_{j=-\infty}^{\infty}\left[\tilde{g}\left(t-j T_{f}\right)\right. \\
& \left.+b_{\left\lfloor j / N_{f}\right\rfloor} \tilde{g}\left(t-j T_{f}-T_{d}\right)\right]+\tilde{n}(t),
\end{aligned}
$$

where $\tilde{g}(t)$ and $\tilde{n}(t)$ are filtered versions of $g(t)$ and $n(t)$, respectively.

\section{A. Conventional TR Receivers}

In conventional TR receiver, the signal $\tilde{r}(t)$ and a delayed version of this signal are correlated, as shown in Fig. 3(a). The outputs of the correlator are summed over the $N_{f}$ frames to acquire the decision statistic. The decision statistic for TR signaling [14] can be written as

$$
Z_{T R}=\sum_{j=0}^{N_{f}-1} \int_{j T_{f}+T_{d}}^{j T_{f}+T_{d}+T_{I}} \tilde{r}(t) \tilde{r}\left(t-T_{d}\right) d t,
$$

where $T_{I}$ is the integration interval in each frame which satisfies $0<T \leq T_{g}$. The bit decision is made using conventional detection as $\hat{b}=\operatorname{sgn}\left(Z_{T R}\right)$, where $\operatorname{sgn}(\cdot)$ stands for the sign function.

The performance of TR system can be improved using ATR shown in Fig. 3(b), which performs averaging over the $N_{f}$ previous reference pulses prior to demodulation, as described in [6], [8]. Fig. 2(b) shows a slightly modified transmitted sequence, which illustrates that all the reference pulses in a bit interval are transmitted first and their corresponding data-modulated pulses follow with a delay $T_{d}=N_{f} T_{f} / 2$. This signaling sequence needs comparatively shorter delays for averaging and also requires that the channel remains invariant only over one bit duration. The averaged template denoted as $g_{\text {(ATR) }}(t)$ is formed over $N_{f}$ reference pulses as,

$$
g_{\text {(ATR) }}(t)=\frac{1}{N_{f}} \sum_{i=0}^{N_{f}-1} \tilde{r}\left(t+i T_{f} / 2\right), \quad t \in\left[0, T_{f} / 2\right] .
$$

The decision statistic is obtained by performing correlation between the averaged estimated template and all the datamodulated pulses within a bit duration.

\section{B. Recursive TR Receivers}

In the proposed R-TR receiver structure, the reference template is estimated in a recursive manner to enable the receiver to capture adequate multipath diversity, see Fig. 4(a). First, the previous estimated template is obtained using a delay $D$, since the template estimation process assumes that the delay between two subsequent reference pulses is $D=T_{f}$, see Fig. 2(a). The previous estimated template and the reference pulse in the newly received frame are pre-multiplied with appropriate weights and then added to obtain new estimated

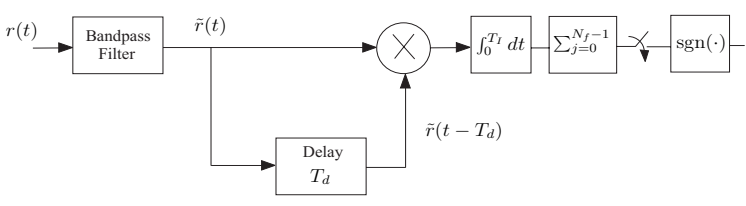

(a)

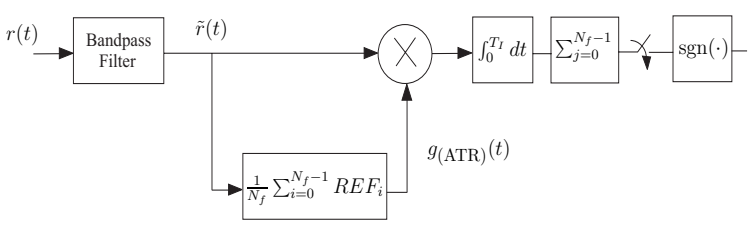

(b)

Fig. 3. (a) Structure of the conventional TR receiver, (b) structure of the conventional ATR receiver ( $R E F$ stands for the reference pulses).

template. Thus, the template update is performed frame-byframe which essentially requires the weighting and alignment of the previous template estimate and the newly received reference pulse. Further, a delayed version of the newly estimated template is obtained by using a delay of $T_{d}$, which is the delay between reference and modulated pulse. The portion of the frame that holds the estimated template is utilized in the correlation operation. The recursion process can be expressed as

$$
g_{(\mathrm{R}-\mathrm{TR})_{k}}(t)=\mu g_{(\mathrm{R}-\mathrm{TR})_{k-1}}(t-D)+\beta \tilde{r}_{k}(t),
$$

where $t \in\left[0, T_{f} / 2\right], g_{(\mathrm{R}-\mathrm{TR})_{k}}(t)$ is the new template and $g_{(\mathrm{R}-\mathrm{TR})} k_{-1}(t-D)$ is the previous template estimates at $k$ th and $(k-1)$ th iteration or frame interval, respectively. The constants $\mu$ and $\beta$ can be considered as the weighting factors of the previous template and the new reference pulse, respectively. The constant $\mu$ can also be interpreted as the forgetting factor of previous template estimates. If the channel is fast timevarying, this factor can help the estimated template to adapt the channel variations. If the channel is assumed to be static, the parameter $\mu=\beta=1 / 2$ can be used, then (9) reduces to recursive averaging as

$$
g_{(\mathrm{R}-\mathrm{TR})_{k}}(t)=\frac{1}{2}\left[g_{(\mathrm{R}-\mathrm{TR})_{k-1}}(t-D)+\tilde{r}_{k}(t)\right] .
$$

The estimated reference template can be written as

$$
g_{(\mathrm{R}-\mathrm{TR})}(t)=\sqrt{E_{p}} g(t)+\hat{n}_{j}(t), \quad t \in\left[0, T_{f} / 2\right],
$$

where $j$ stands for the $j$ th template, $E_{p}$ is the transmitted energy per pulse, $g(t)$ is the clean template, and $\hat{n}_{j}(t)$ is the residual noise in the estimated template. This estimated template waveform is used to perform correlation with the received signal. The output of multiplier of the correlator consists of signal regions and noise-only regions, so the choice of integration interval becomes a crucial parameter as the integration should be performed only over the signal regions. The selection of an optimum integration interval can avoid the accumulation of noise in the decision variable. In this case, the decision statistic at the output of the correlator assuming an ideal synchronization is given by, 


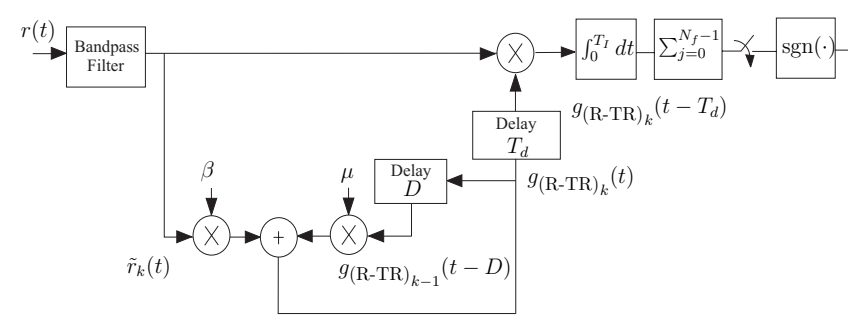

(a)

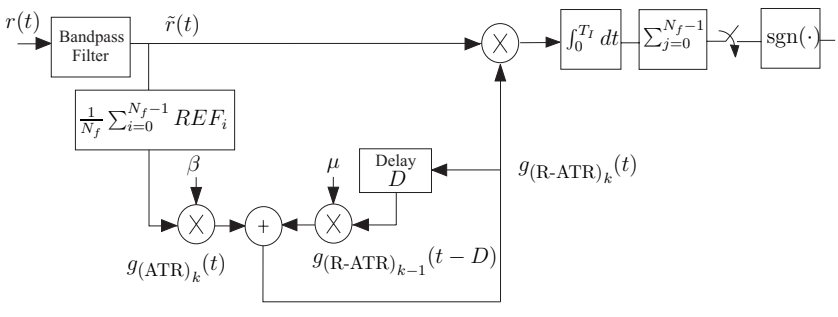

(b)

Fig. 4. (a) Structure of the R-TR receiver, (b) structure of the R-ATR receiver ( $R E F$ stands for the reference pulses).

$$
\begin{aligned}
Z_{R-T R}= & \sum_{j=0}^{N_{f}-1} \int_{j T_{f}+T_{d}}^{j T_{f}+T_{d}+T_{I}} \tilde{r}(t) g_{(\mathrm{R}-\mathrm{TR})}\left(t-T_{d}\right) d t \\
= & \sum_{j=0}^{N_{f}-1} \int_{j T_{f}+T_{d}}^{j T_{f}+T_{d}+T_{I}}\left(\sqrt{E_{p}} b \tilde{g}_{j}(t)+\tilde{n}_{j}(t)\right) \\
& \cdot\left(\sqrt{E_{p}} g\left(t-T_{d}\right)+\hat{n}_{j}\left(t-T_{d}\right)\right) d t \\
= & \sum_{j=0}^{N_{f}-1} \int_{j T_{f}+T_{d}}^{j T_{f}+T_{d}+T_{I}}\left[E_{p} b_{k} \tilde{g}_{j}(t) g\left(t-T_{d}\right)\right. \\
& +\sqrt{E_{p}} b \tilde{g}_{j}(t) \hat{n}_{j}\left(t-T_{d}\right)+\sqrt{E_{p}} g\left(t-T_{d}\right) \tilde{n}_{j}(t) \\
& \left.+\tilde{n}_{j}(t) \hat{n}_{j}\left(t-T_{d}\right)\right] d t .
\end{aligned}
$$

The signal component in (12) can be approximated as $N_{f} E_{p} b \boldsymbol{\alpha}^{2} R(0)$, where $\boldsymbol{\alpha}=\left[\alpha_{0}, \alpha_{1}, \ldots, \alpha_{L-1}\right] \quad$ and $R(\tau)=\int_{-\infty}^{\infty} p(t) p(t-\tau) d t$. The noise $\tilde{n}_{j}(t)$ and $\hat{n}_{j}(t)$ are zero mean Gaussian independent of $\tilde{g}_{j}(t)$, so the noise-signal terms in (12) can be considered as zero mean Gaussian random variables. As in [8], the noise-noise term is sum of $N_{f}$ independent random variables each of the form $\int_{j T_{f}+T_{d}}^{j T_{f}+T_{d}+T_{I}} \tilde{n}_{j}(t) \hat{n}_{j}\left(t-T_{d}\right) d t$, for different values of $j$. This term can also be approximated as a zero mean Gaussian random variable according to the central-limit theorem.

Furthermore, the conventional noise averaging ATR scheme is extended to generate a recursive structure called recursive averaged TR (R-ATR) scheme, as depicted in Fig. 4(b). First, like ATR, the proposed R-ATR receiver performs average by appropriately delaying $N_{f}$ received reference pulses within the current bit, see Fig. 2(b). In addition, the resulting waveform and the previously estimated template are pre-multiplied with appropriate weights and then added to obtain the new reference template. The previous template estimate is kept in a memory with delay $D=N_{f} T_{f}$ to be utilized in the recursion process. It is noteworthy that the template update is performed bit-by-bit in this case, which means that there is only a slight increase in complexity. In a similar fashion as (9), the template estimation process can be expressed as

$$
g_{(\mathrm{R}-\mathrm{ATR})_{k}}(t)=\mu g_{(\mathrm{R}-\mathrm{ATR})_{k-1}}(t-D)+\beta g_{(\mathrm{ATR})_{k}}(t),
$$

where $g_{(\mathrm{R}-\mathrm{ATR})_{k}}(t)$ is the new template and $g_{(\mathrm{R}-\mathrm{ATR})_{k-1}}(t-D)$ is the previous template estimates at $k$ th and $(k-1)$ th iteration or bit interval, respectively. The term $g_{(\mathrm{ATR})}(t)$ represents the average over $N_{f}$ received reference pulses within the current bit duration. Again, the parameter $\mu=\beta=1 / 2$ can be used assuming that the channel is static, then (13) reduces to recursive averaging as

$$
g_{(\mathrm{R}-\mathrm{ATR})_{k}}(t)=\frac{1}{2}\left[g_{(\mathrm{R}-\mathrm{ATR})_{k-1}}(t-D)+g_{(\mathrm{ATR})}(t)\right] .
$$

In case of R-ATR scheme, the newly estimated template $g_{(\mathrm{R}-\mathrm{ATR})_{k}}(t)$ is used in the correlator for demodulation of all the subsequent data-modulated pulses over a bit interval to form the decision statistic.

The IR-UWB systems use time-hopping (TH) and polarity scrambling to obtain processing gain, to combat multipleaccess interference (MAI), and to smooth the signal spectrum [15]. The recursive schemes described above assume that $\mathrm{TH}$ is absent. If $\mathrm{TH}$ is used in conjunction with TR signaling, averaging of the template estimates in the R-TR scheme should be performed by appropriately delaying the estimated template from the previous frame using the TH sequence. The complexity of the proposed schemes can be reduced substantially by repeating the TH sequence after a bit duration. In this case, the recursive averaging process in R-TR scheme can be accomplished by delaying the corresponding estimated templates from the previous bit instead to use a fixed delay element. Similarly, the recursion process in R-ATR scheme also requires only a fixed delay element in this case. Further, the receiver frontend of both ATR and R-ATR schemes is the same with the slightly modified transmitted sequence shown in Fig. 2(b). However, if pulse-based polarity scrambling is used with ATR and R-ATR schemes, the descrambling of the polarity should be performed in the receiver before the averaging operations.

\section{Performance Evaluation}

In order to evaluate performance of the receivers, TR IRUWB system is simulated on the multipath channels proposed by IEEE $802.15 .4 a$ for low-data-rate UWB systems. The binary antipodal modulation is used employing second derivative of the Gaussian pulse with about $1.4 \mathrm{~ns}$ pulse duration. The uncoded data rate of $0.5 \mathrm{Mbps}$ is achieved with $N_{f}=10$ and $T_{f}=200 \mathrm{~ns}$. The delay $T_{d}=100 \mathrm{~ns}$ and the integration interval within each frame is $T_{I}=50 \mathrm{~ns}$. The channel model CM1 is used which covers residential line-of-sight (LOS) environments with maximum delay spread of about $100 \mathrm{~ns}$, 


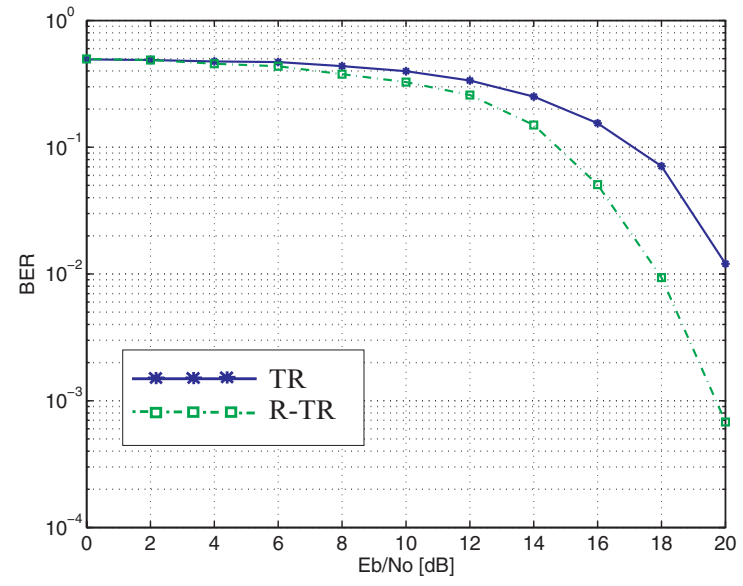

Fig. 5. Uncoded BER curves of TR and R-TR receivers over CM1 channel.

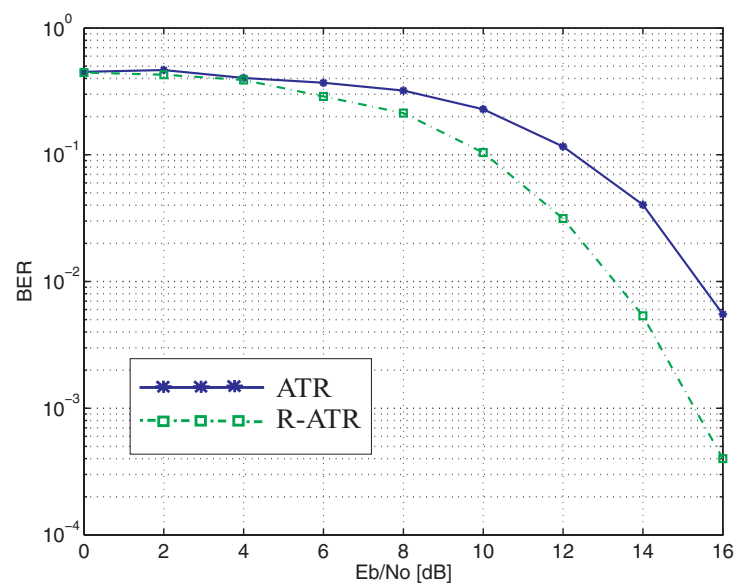

Fig. 6. Uncoded BER curves of ATR and R-ATR receivers over CM1 channel.

which means that a negligible IFI occurs. The realistic indoor UWB channels usually have long channel coherence time, so the channel coherence time for the channel is assumed to be $0.2 \mathrm{~ms}$ and the parameters of the recursive averaging process are set as $\mu=\beta=1 / 2$. The energy of the channel impulse responses is normalized as $\sum \alpha_{l}^{2}=1$ and the system is assumed to be perfectly synchronized.

The simulated uncoded BER curves are shown in Fig. 5 for TR and R-TR receiver. The results validate that the R-TR structure has about $2 \mathrm{~dB}$ better performance than the conventional $\mathrm{TR}$ receiver. It is worth mentioning that in high SNR region, the effect of multipath is more dominant, so increasing the length of the integration interval is more effective to improve the BER. Similarly, Fig. 6 shows BER comparison curves for ATR and R-ATR approaches employing the same parameters. The BER plots depict that the R-ATR yields a similar gain in SNR over its simple noise averaging counterpart, i.e., ATR receiver. It can be concluded that the recursive averaging process helps to further alleviate the noise effect in the estimated template, which in turn improves BER performance.

\section{CONCLusions}

This paper presents recursive schemes of the transmitted reference receiver structure for the detection of low-data-rate impulse radio UWB signals. The proposed recursive schemes estimate the reference template for the correlator recursively by appropriately weighting and aligning the received reference pulses and the previous estimated templates. In case of timehopping, the complexity of the recursion process can be significantly reduced by repeating the time-hopping sequence after a bit duration to use a bit long fixed delay. The performance evaluation of the TR IR-UWB system indicates that the R-TR and R-ATR receiver schemes outperform the conventional TR and ATR receiver over slowly-varying channels. The use of slightly modified TR signaling sequence in conjunction with R-ATR receiver scheme is especially of interest as it can yield an improved performance with only a slight increase in complexity.

\section{REFERENCES}

[1] M.-G. di Benedetto, T. Kaiser and N. Schmidt UWB - State of the Art, Journal of Applied Signal Processing Editorial, EURASIP publishing, March 2005

[2] J. G. Proakis Digital Communications, 4th ed. Boston: McGraw-Hill, 2001.

[3] M. G. Khan, J. Nordberg, A. Mohammed, and I. Claesson, "Performance evaluation of RAKE receiver for UWB systems using measured channels in industrial environments," AusWireless'06, Int. Conf. on Wireless Broadband and Ultrawideband Systems, March 2006.

[4] D. Cassioli, M. Z. Win, F. Vatalaro, and A. F. Molisch, "Performance of low-complexity Rake reception in a realistic UWB channel," Proc. IEEE ICC'03, vol.2, pp.763-767, 2003.

[5] F. Tufvesson, A. F. Molisch, "Ultra-wideband communication using hybrid matched filter correlation receivers," Proc. IEEE Veh. Technol. Conf., vol. 3, pp. 1290-1294, May 2004.

[6] D. J. Choi, W. E. Stark "Performance of ultra-wideband communications with suboptimal receivers in multipath channels,"IEEE Journal of Sleceted Areas Comm., vol. 20, no. 9, Dec. 2002, 1754-1766.

[7] R. T. Hoctor and H. W. Tomlinson, "An overviewof delay-hopped, transmitted- reference RF communications," in Technical Information Series: G.E. Research and Development Center, Jan. 2002, pp. 129.

[8] Y.-L. Chao, R. A. Scholtz "Optimal and suboptimal receivers for ultra-wideband transmitted reference systems,"IEEE Global Telecommun. Conf.'03, vol. 2, pp. 759-763, 1-5 Dec. 2003

[9] J. Romme, K. Witrisal, "Transmitted-reference UWB systems using weighted autocorrelation receivers,"IEEE Trans. on Microwave Theory and Tech., vol. 54, no. 4, pp. 1754-1761, April 2006.

[10] G. Durisi, S. Benedetto, "Comparison between coherent and noncoherent receivers for UWB Communications," UWB - State of the Art, Journal of Applied Signal Processing Editorial, EURASIP

[11] S. Zhao, H. Liu, Z. Tian, "Decision directed autocorrelation receivers for pulsed ultra-wideband systems,"IEEE Trans. Wireless Commun., vol. 5, no. 8, pp. 2175-2184, Aug. 2006.

[12] A. Saleh and R. Valenzuela, "A statistical model for indoor multipath propagation," IEEE Journal on Select. Areas Commun., vol. SAC-5, no. 2, pp. 128-137, Feb. 1987.

[13] A. F. Molisch et al., "IEEE 802.15.4a channel model - final report," Tech. Rep. Document IEEE 802.15-04-0662-02-004a, 2005.

[14] T. Q. S. Quek, M. Z. Win and D. Dardari, UWB transmitted reference signaling schemes - part I: performance analysis, Proc. IEEE Conference ICU 2005., pp. 587- 592, Sep. 2005.

[15] S. Zhao, P. Orlik, A. F. Molisch, H. Liu, J. Zhang "Hybrid Ultrawideband Modulations Compatible for Both Coherent and Transmit-Reference Receivers,"IEEE Trans. on Wireless Commun. 